\title{
Bi-Attempted Based Optimization Algorithm For Numerical Optimization Problems
}

\author{
Mehtap Köse Ulukök ${ }^{1 *}$

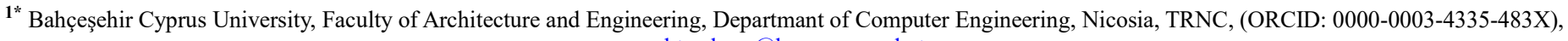 \\ mehtap.kose@baucyprus.edu.tr
}

(3rd International Congress on Human-Computer Interaction, Optimization and Robotic Applications June 11-13, 2021)

(DOI: 10.31590/ejosat.953349)

ATIF/REFERENCE: Köse Ulukök, M. (2021). Bi-Attempted Based Optimization Algorithm For Numerical Optimization Problems. European Journal of Science and Technology, (26), 466-471.

\begin{abstract}
Numerical optimization is one of the well-known problems in computer science. Day by day, new methods are developed by many researchers. Recently, optimization became an essential task for many disciplines, such as engineering, medicine, management and others. In many cases, optimization problems may require fast and efficient algorithms for real-time implementations. In this paper, a simple, fast and feasible algorithm is presented for the optimization of both uni-modal and multi-modal benchmark functions. A population based Bi-Attempted Based Optimization Algorithm (ABaOA) is a stochastic search method which searches a solution space with two fixed step-size displacement parameters and two mutation operators. The proposed algorithm is derived from Base Optimization Algorithm (BaOA) which uses basic arithmetic operations. The performance of ABaOA is tested on twenty well-known benchmark functions and the results are statistically compared with the seven well-known stochastic optimization algorithms. Three different statistical analyses were done on the results obtained from the ABaOA. Two non-parametric statistical comparisons with the mean values are performed by using Sign and Wilcoxon tests. The non-parametric statistical multiple comparisons of the proposed algorithm is performed by using the Friedman test. The non-parametric Friedman test of differences among repeated measures of these algorithms was conducted and referred a Chi-square value of 67.337, which was significant ( $\mathrm{p}<0.05)$. Wilcoxon non-parametric pairwise comparison test was applied to analyze the difference of $\mathrm{ABaOA}$ statistically among the other algorithms. The test indicates that the introduced algorithm is statistically significant than other algorithms with a level of significance $\mathrm{p}<0.05$. The experimental results also show that the $\mathrm{ABaOA}$ is clearly superior to the compared stochastic optimization algorithms.
\end{abstract}

Keywords: Computational Intelligence, Evolutionary Computation, Optimization Methods, Numerical Optimization.

\section{Sayısal İyileştirme Problemleri İçin İki-Aşamalı Temel İyileştirme Algoritması}

$\ddot{O} \mathbf{z}$

Sayısal iyileştirme, bilgisayar biliminde iyi bilinen sorunlardan biridir. Gün geçtikçe birçok araştırmacı tarafından yeni yöntemler geliştirilmektedir. Son zamanlarda iyileştirme yapmak, mühendislik, tıp, yönetim ve diğerleri gibi birçok disiplin için önemli bir zorunluluk haline geldi. Çoğu durumda, iyileştirme problemleri, gerçek zamanlı uygulamalar için hızlı ve verimli algoritmalar gerektirebilir. $\mathrm{Bu}$ yazıda, hem tek-modelli hem de çoklu-modelli kıyaslama fonksiyonlarının iyileştirilmesi için basit, hızlı ve uygulanabilir bir algoritma sunulmuştur. Popülasyon tabanlı Bi-Attempted Base Optimizasyon Algoritması (ABaOA), iki sabit adım kaydırma parametresi ve iki mutasyon operatörü ile bir çözüm alanını arayan stokastik bir arama yöntemidir. Önerilen algoritma, temel aritmetik işlemleri kullanan Base Optimizasyon Algoritmasından (BaOA) türetilmiştir. ABaOA'nın performansı, iyi bilinen yirmi kıyaslama fonksiyonu üzerinde test edilmiş ve sonuçlar, iyi bilinen yedi stokastik optimizasyon algoritması ile istatistiksel olarak

$1^{*}$ Corresponding Author: mehtap.kose@baucyprus.edu.tr 
karşılaştırılmıştır. ABaOA'dan elde edilen sonuçlar üzerinde üç farklı istatistiksel analiz yapılmıştır. Sign ve Wilcoxon testleri kullanılarak ortalama değerlerle iki parametrik olmayan istatistiksel karşılaştırma yapılmıştır. Önerilen algoritmanın parametrik olmayan istatistiksel çoklu karşılaştırması Friedman testi kullanılarak gerçekleştirilmiştir. Bu algoritmaların tekrarlanan ölçümleri arasındaki farklılıkların parametrik olmayan Friedman testi 67.337'lik bir Ki-kare değeri istatistiksel olarak anlamlı bulundu (p $<0.05$ ). ABaOA'nın diğer algoritmalar arasındaki farkını istatistiksel olarak analiz etmek için Wilcoxon parametrik olmayan ikili karşılaştırma testi uygulanmıştır. Test, sunulan algoritmanın diğer algoritmalardan istatistiksel olarak anlamlı olduğunu ve p <0,05 anlamlılık düzeyine sahip olduğunu göstermektedir. Deneysel sonuçlar ayrıca ABaOA'nın karşılaştırılan stokastik iyileştirme algoritmalarından açıkça üstün olduğunu göstermektedir

Anahtar Kelimeler: Sayısal Zeka, Evrimsel Hesaplama, İyileştirme Yöntemleri, Sayısal İyileştirme.

\section{Introduction}

Limited resources left in all over the world. This is why it is very important to consume them in more efficient way. The consumption of water, energy and other materials should be carefully decided. Therefore, optimization is an indispensable task for many disciplines specially for various engineering problems (Campbell et al., 2019; de Melo \& Banzhaf, 2018). Naturally inspired algorithms such as Genetic Algorithms (GA) (Holland, 1962), Particle Swarm Optimization (PSO) (Deb \& Padhye, 2014), Ant Colony Optimization (ACO) (Dorigo \& Blum, 2005), Artificial Bee Colonoy (ABC) (Karaboga \& Akay, 2009), and the others can be used for both numerical and combinatorial optimization problems (Song et al., 2019). On the other hand, Branch-and-Bound, DIRECT, gradient-based optimization, sequential quadratic programming (Bednár et al., 2019; Liberti \& Kucherenko, 2005) are some of the deterministic approaches for numerical optimization problems. Optimization algorithms are frequently modified or altered to increase their performances for a specific optimization problem (Cao et al., 2019; Zang et al., 2018). All optimization algorithms; mainly metaheuristics have some number of parameters to be set, and they require long computation time. The quality of solutions which are derived from metaheuristics is highly depended on their parameter settings. On the other hand, deterministic algorithms may require less number of parameters, and they may find the desired solution in more quicker time than the metaheuristic ones. Deterministic algorithms may be better to use for some real-life problems because of their superior performance (Bednár et al., 2019; Liberti \& Kucherenko, 2005).

The main reason for the wide variety of metaheuristic algorithms is the drawback of parameter settings and their computational complexity. Therefore, algorithms that require less number of parameter settings and have self-adaptive abilities become the interest of many studies (Yadav et al., 2020). Moreover, there are also some metaheuristic algorithms that are inspired from natural life. These algorithms aim to mimic the power of natural evolution in the optimization problems.

In this paper, a population based Bi-Attempted Based Optimization Algorithm ( $\mathrm{ABaOA})$ is introduced which is derived from Base Optimization Algorithm (BaOA) with two fixed displacement parameters and two mutation operators. $\mathrm{BaOA}$ is reported as fast and feasible algorithm for numerical optimization (Xing \& Gao, 2014).

\section{Material and Method}

\subsection{Bi-Attempted Based Optimization Algoritm}

The Base Optimization Algorithm (BaOA) has been inspired from mathematics. BaOA simply applies arithmetic operators to a potential solution. It has only one parameter $\delta$ as a displacement parameter and two range constants as Rmin and Rmax, respectively. By tuning the value of one displacement parameter, the performance of the $\mathrm{BaOA}$ algorithm can be improved (Xing $\&$ Gao, 2014). The BaOA starts with randomly generated potential solutions, and they updated based on four arithmetic operators. Best found solution so far is kept in each iteration until the desired number of iterations has been performed.

The quick convergence and simplicity of $\mathrm{BaOA}$ are preserved and improved by adding one more displacement variable and by reducing the four arithmetic operators to two. The use of only two arithmetic operators; addition and subtraction operations, as a mutation operator leads to decrease the computation time of $\mathrm{BaOA}$. Moreover, the use of two different displacement variables preserves the quick convergence behaviour and the solution quality of the algorithm. The modified algorithm is called as BiAttempted Base Optimization Algorithm (ABaOA) and it is given in Table 1 .

Metaheuristic algorithms use global and local search techniques to reach a global optimum point in the search space. These techniques have high computation time and programming complexity. Finding a feasible solution by these algorithms depends on probabilistic approaches in its nature. However, deterministic algorithms based on exact models and they guarantee to reach a global optimum. The two different displacement parameters in $\mathrm{ABaOA}$ mimic the global and local search techniques in its search process.

\section{Table 1: Bi-Attempted Base Optimization Algorithm} $(\mathrm{ABaOA})$

- $\quad$ Generate $\mathrm{n}$ random potential solutions (S1 ... Sn) with d dimensions

- While maximum number of iterations has been reached

- For each potential solution $\mathrm{Si}$

- Calculate fitness value of each $\mathrm{f}(\mathrm{Si})$

- Derive new potential solutions as:

$$
\left.\begin{array}{l}
S_{i, 1}^{+}[i]=S_{i}[i]+\delta_{1} \\
S_{i, 2}^{+}[i]=S_{i}[i]+\delta_{2} \\
S_{i, 1}^{-}[i]=S_{i}[i]-\delta_{1} \\
S_{i, 2}^{-}[i]=S_{i}[i]-\delta_{2}
\end{array}\right\} R_{\min } \leq S_{i}^{*}[j] \leq R_{\max }
$$

- Compute fitness values of new potential solutions $f_{i, 1}^{+}, f_{i, 1}^{-}, f_{i, 2}^{+}, f_{i, 2}^{-}$

- Update fitness value of each $\mathrm{f}(\mathrm{Si})$ with the best

$$
f\left(S_{i}\right)=\min \left\{f_{i, 1}^{+}, f_{i, 1}^{-}, f_{i, 2}^{+}, f_{i, 2}^{-}\right\}
$$


where, $f$ is the test function, $S_{i}$ is the potential solution, $R_{\min }$ and $R_{\max }$ are the minimum and maximum ranges, respectively. The two displacement parameters $\delta_{1}$ and $\delta_{2}$ preserve the quick convergence to the global optimum point of $\mathrm{ABaOA}$.

\subsubsection{Benchmark Functions}

The $\mathrm{ABaOA}$ is tested on twenty well-known benchmark functions. These benchmark functions are chosen as same with the study given in (Chakri et al., 2017) and the obtained results are compared with those. These functions' name, domain and feasible solutions are summarized in Table 2.

Table 2: Used benchmark numerical functions's domain and feasible solutions

\begin{tabular}{|c|c|c|c|}
\hline & Function name & Domain & Feasible Solution \\
\hline 1 & Sphere & $x_{i} \in[-5.12,5.12]$ & $\mathbf{x}^{*}=(0,0, \ldots, 0) ; f_{1}\left(\mathbf{x}^{*}\right)=0$ \\
\hline 2 & $\begin{array}{l}\text { Sum of Different } \\
\text { Powers }\end{array}$ & $x_{i} \in[-100,100]$ & $\mathbf{x}^{*}=(0,0, \ldots, 0) ; f_{2}\left(\mathbf{x}^{*}\right)=0$ \\
\hline 3 & Rotated Hyper-ellipsoid & $x_{i} \in[-65,65]$ & $\mathbf{x}^{*}=(0,0, \ldots, 0) ; f_{3}\left(\mathbf{x}^{*}\right)=0$ \\
\hline 4 & Griewank & $x_{i} \in[-600,600]$ & $\mathbf{x}^{*}=(0,0, \ldots, 0) ; f_{4}\left(\mathbf{x}^{*}\right)=0$ \\
\hline 5 & Trid & $x_{i} \in\left[-d^{2}, d^{2}\right]$ & $\begin{array}{l}\mathbf{x}^{*}=i(d+1-i), i=1,2 \ldots d \\
f_{5}\left(\mathbf{x}^{*}\right)=-d(d+4)(d-1) / 6\end{array}$ \\
\hline 6 & Rastrigin & $x_{i} \in[-5.12,5.12]$ & $\mathbf{x}^{*}=(0,0, \ldots, 0) ; f_{6}\left(\mathbf{x}^{*}\right)=0$ \\
\hline 7 & Levy & $x_{i} \in[-5.12,5.12]$ & $\mathbf{x}^{*}=(1,1, \ldots, 1) ; f_{7}\left(\mathbf{x}^{*}\right)=0$ \\
\hline 8 & Ackley & $x_{i} \in[-32,32]$ & $\mathbf{x}^{*}=(0,0, \ldots, 0) ; f_{8}\left(\mathbf{x}^{*}\right)=0$ \\
\hline 9 & Schwefel & $x_{i} \in[-500,500]$ & $\mathbf{x}^{*}=(1,1 \ldots, 1) ; f_{9}\left(\mathbf{x}^{*}\right)=0$ \\
\hline 10 & Rosenbrock & $x_{i} \in[-2.048,2.048]$ & $\mathbf{x}^{*}=(1,1 \ldots, 1) ; f_{10}\left(\mathbf{x}^{*}\right)=0$ \\
\hline 11 & Zakharov & $x_{i} \in[-5,10]$ & $\mathbf{x}^{*}=(0,0, \ldots, 0) ; f_{11}\left(\mathbf{x}^{*}\right)=0$ \\
\hline 12 & Dixon-Price & $x_{i} \in[-10,10]$ & $\boldsymbol{x}_{\boldsymbol{i}}=2^{-\frac{2^{i}-2}{2^{i}}}, i=1, \ldots, d ; f_{12}\left(\mathbf{x}^{*}\right)=0$ \\
\hline 13 & Michalewicz & $x_{i} \in[0, \pi]$ & at $d=10 ; f_{13}\left(\mathbf{x}^{*}\right)=-9.66015$ \\
\hline 14 & Powell & $x_{i} \in[-10,10]$ & $\mathbf{x}^{*}=(0,0, \ldots, 0) ; f_{14}\left(\mathbf{x}^{*}\right)=0$ \\
\hline 15 & Bent Cigar & $x_{i} \in[-10,10]$ & $\mathbf{x}^{*}=(0,0, \ldots, 0) ; f_{15}\left(\mathbf{x}^{*}\right)=0$ \\
\hline 16 & Alpine & $x_{i} \in[-10,10]$ & $\mathbf{x}^{*}=(0,0, \ldots, 0) ; f_{16}\left(\mathbf{x}^{*}\right)=0$ \\
\hline 17 & Weierstrass & $x_{i} \in[-0.9,0.9]$ & $C E C^{\prime} 15, f_{17}\left(\mathbf{x}^{*}\right)=300$ \\
\hline 18 & Stybinlinski-Tang & $x_{i} \in[-10,10]$ & $x^{*}=(-2,903534, \ldots,-2,903534) ; f_{18}\left(x^{*}\right)=0$ \\
\hline 19 & Salomon & $x_{i} \in[-100,100]$ & $\mathbf{x}^{*}=(0,0, \ldots, 0) ; f_{19}\left(\mathbf{x}^{*}\right)=0$ \\
\hline 20 & Shaffer F7 & $x_{i} \in[-100,100]$ & $\mathbf{x}^{*}=(0,0, \ldots, 0) ; f_{20}\left(\mathbf{x}^{*}\right)=0$ \\
\hline
\end{tabular}

\section{Results and Discussion}

The results of the ABaOA on twenty benchmark function are compared with seven different heuristics and the BaOA. Best, median, worst, mean and standard deviation of $\mathrm{ABaOA}, \mathrm{BaOA}$ over 51 runs are recorded together with the results reported in (Chakri et al., 2017) and they are summarized in Table 3 and Table 4.

In order to proof whether the $\mathrm{ABaOA}$ statistically significant than the other algorithms, two non-parametric statistical comparisons with the mean values are done by using Sign and Wilcoxon tests. The non-parametric statistical multiple comparison of algorithms is performed by using the Friedman test, and the results are given in Table 5.

The non-parametric Friedman test of differences among repeated measures of these algorithms was conducted and referred a Chi-square value of 67.337 which was significant $(p<0.05)$. The Wilcoxon non-parametric pairwise comparison test is applied to analyze the difference of $\mathrm{ABaOA}$ statistically among the other algorithms. The test indicates that the introduced algorithm is statistically significant than other algorithms with a level of significance $\mathrm{p}<0.05$. The Wilcoxon non-parametric pairwise comparison test and the sign test statistical results are given in Table 6.

Table 5: Nonparametric statistical multiple comparison results of $\mathrm{ABaOA}$

\begin{tabular}{l|l}
\hline \multicolumn{1}{c|}{ Algorithm } & \multicolumn{1}{c}{ Friedman mean Rank } \\
\hline$A B a O A$ & 1.97 \\
\hline$B a O A$ & 3.82 \\
\hline$d B A$ & 3.32 \\
\hline$B A$ & 6.95 \\
\hline$P S O$ & 7.42 \\
\hline$H S$ & 6.79 \\
\hline$C S$ & 5.21 \\
\hline$D E$ & 5.00 \\
\hline$G A$ & 4.53 \\
\hline \multicolumn{2}{r}{} \\
\hline
\end{tabular}


Table 3: Comparison between ABaOA and classical algorithm on benchmark function (F1 - F10)

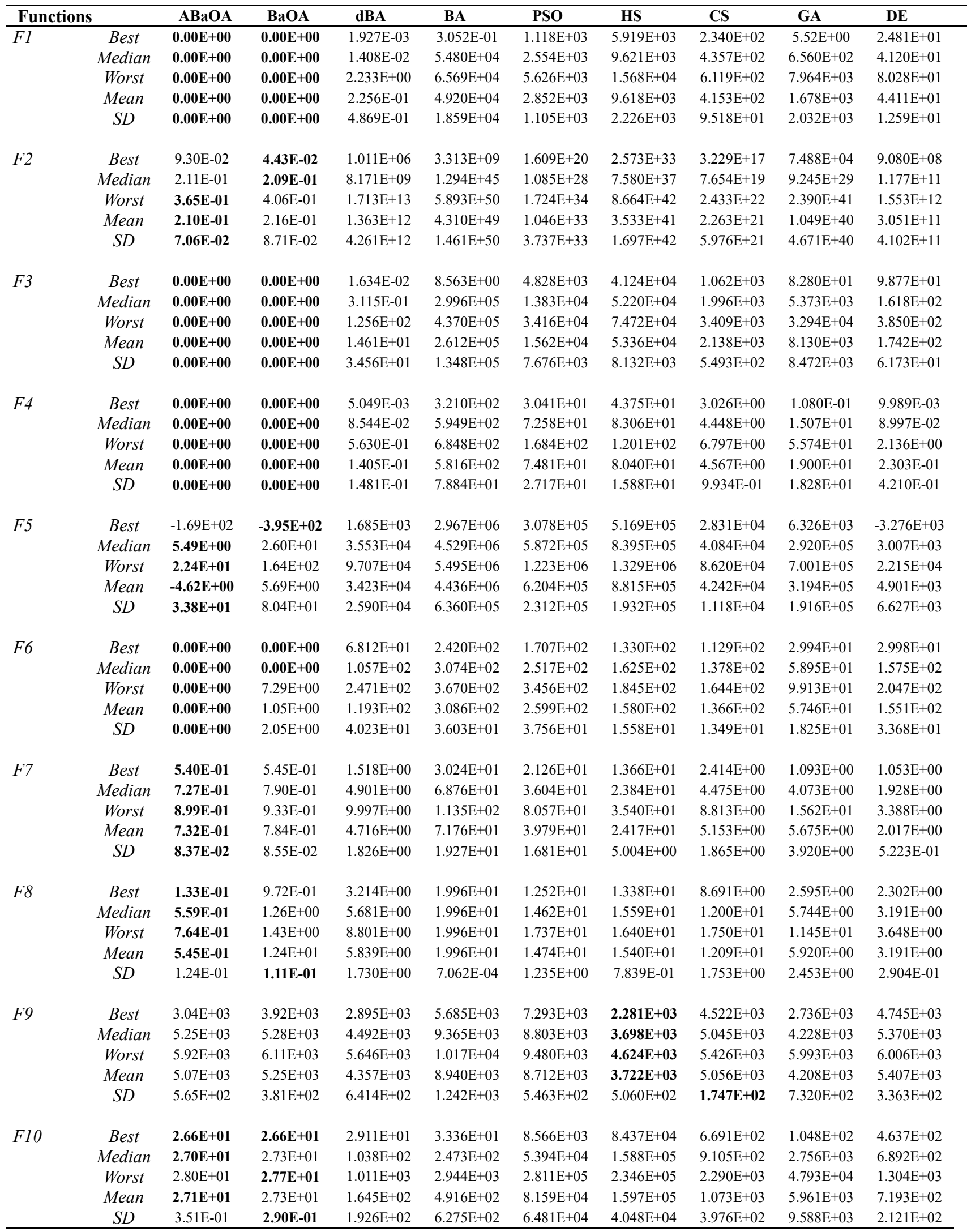


European Journal of Science and Technology

Table 4: Comparison between ABaOA and classical algorithm on benchmark function (F10 - F20)

\begin{tabular}{|c|c|c|c|c|c|c|c|c|c|c|}
\hline \multicolumn{2}{|c|}{ Functions } & ABaOA & BaOA & dBA & BA & PSO & HS & CS & GA & DE \\
\hline \multirow[t]{5}{*}{$F 11$} & Best & $5.54 \mathrm{E}-03$ & $2.03 \mathrm{E}+01$ & $7.536 \mathrm{E}+01$ & $4.799 \mathrm{E}+00$ & $5.754 \mathrm{E}+02$ & $2.960 \mathrm{E}+02$ & $1.337 \mathrm{E}+02$ & $1.112 \mathrm{E}+01$ & $1.414 \mathrm{E}+02$ \\
\hline & Median & 2.03E-02 & $5.98 \mathrm{E}+01$ & $1.561 \mathrm{E}+02$ & $3.103 \mathrm{E}+01$ & $1.108 \mathrm{E}+03$ & $4.023 \mathrm{E}+02$ & $2.190 \mathrm{E}+02$ & $7.707 \mathrm{E}+06$ & $1.879 \mathrm{E}+02$ \\
\hline & Worst & 3.12E-02 & $9.84 \mathrm{E}+01$ & $2.506 \mathrm{E}+02$ & $1.334 \mathrm{E}+02$ & $1.616 \mathrm{E}+03$ & $5.713 \mathrm{E}+02$ & $3.009 \mathrm{E}+02$ & $9.316 \mathrm{E}+08$ & $2.352 \mathrm{E}+02$ \\
\hline & Mean & 2.02E-02 & $5.96 \mathrm{E}+01$ & $1.515 \mathrm{E}+02$ & $4.629 \mathrm{E}+01$ & $1.054 \mathrm{E}+03$ & $4.052 \mathrm{E}+02$ & $2.214 \mathrm{E}+02$ & $9.884 \mathrm{E}+07$ & $1.937 \mathrm{E}+02$ \\
\hline & $S D$ & $5.89 \mathrm{E}-03$ & $1.54 \mathrm{E}+01$ & $4.105 \mathrm{E}+01$ & $3.971 \mathrm{E}+01$ & $2.706 \mathrm{E}+02$ & $6.898 \mathrm{E}+01$ & $4.094 \mathrm{E}+01$ & $2.076 \mathrm{E}+08$ & $2.433 \mathrm{E}+01$ \\
\hline \multirow[t]{5}{*}{$F 12$} & Best & $5.76 \mathrm{E}-02$ & $3.60 \mathrm{E}-02$ & 7.448E-01 & $1.323 \mathrm{E}+00$ & $6.914 \mathrm{E}+03$ & $4.057 \mathrm{E}+04$ & $1.059 \mathrm{E}+02$ & $1.207 \mathrm{E}+01$ & $2.650 \mathrm{E}+01$ \\
\hline & Median & $1.50 \mathrm{E}-01$ & $2.22 \mathrm{E}-01$ & $5.528 \mathrm{E}+00$ & $2.187 \mathrm{E}+01$ & $3.195 \mathrm{E}+04$ & $7.688 \mathrm{E}+04$ & $2.200 \mathrm{E}+02$ & $1.836 \mathrm{E}+03$ & $6.164 \mathrm{E}+01$ \\
\hline & Worst & 4.22E- 01 & 5.13E-01 & $1.044 \mathrm{E}+02$ & $9.385 \mathrm{E}+02$ & $1.202 \mathrm{E}+05$ & $1.282 \mathrm{E}+05$ & $6.159 \mathrm{E}+02$ & $3.631 \mathrm{E}+04$ & $1.438 \mathrm{E}+02$ \\
\hline & Mean & $1.65 E-01$ & $2.44 \mathrm{E}-01$ & $1.911 \mathrm{E}+01$ & $1.181 \mathrm{E}+02$ & $3.864 \mathrm{E}+04$ & $7.853 \mathrm{E}+04$ & $2.611 \mathrm{E}+02$ & $6.494 \mathrm{E}+03$ & $6.790 \mathrm{E}+01$ \\
\hline & $S D$ & 7.69E-02 & 1.33E-01 & $2.917 \mathrm{E}+01$ & $2.293 \mathrm{E}+02$ & $2.495 \mathrm{E}+04$ & $2.813 \mathrm{E}+04$ & $1.384 \mathrm{E}+02$ & $9.520 \mathrm{E}+03$ & $2.559 \mathrm{E}+01$ \\
\hline \multirow[t]{5}{*}{ F13 } & Best & $-1.29 E+01$ & $-9.26 \mathrm{E}+00$ & $-2.094 \mathrm{E}+01$ & $-9.637 \mathrm{E}+00$ & $-1.307 \mathrm{E}+01$ & $-1.477 \mathrm{E}+01$ & $-1.673 \mathrm{E}+01$ & $-2.473 \mathrm{E}+01$ & $-1.259 \mathrm{E}+01$ \\
\hline & Median & $-1.18 E+01$ & $-7.44 \mathrm{E}+00$ & $-1.470 \mathrm{E}+01$ & $-8.037 \mathrm{E}+00$ & $-9.785 E+00$ & $-1.396 \mathrm{E}+01$ & $-1.444 \mathrm{E}+01$ & $-2.154 \mathrm{E}+01$ & $-1.123 \mathrm{E}+01$ \\
\hline & Worst & $-1.06 E+01$ & $-6.53 E+00$ & $-1.017 \mathrm{E}+01$ & $-6.880 \mathrm{E}+00$ & $-7.011 \mathrm{E}+00$ & $-1.208 \mathrm{E}+01$ & $-1.333 \mathrm{E}+01$ & $-1.871 \mathrm{E}+01$ & $-1.011 \mathrm{E}+01$ \\
\hline & Mean & $-1.17 E+01$ & $-7.58 \mathrm{E}+00$ & $-1.495 \mathrm{E}+01$ & $-8.179 \mathrm{E}+00$ & $-9.620 \mathrm{E}+00$ & $-1.376 \mathrm{E}+01$ & $-1.455 \mathrm{E}+01$ & $-2.186 \mathrm{E}+01$ & $-1.121 \mathrm{E}+01$ \\
\hline & $S D$ & $5.94 \mathrm{E}-01$ & $5.99 \mathrm{E}-01$ & $3.135 \mathrm{E}+00$ & $6.847 \mathrm{E}-01$ & $1.732 \mathrm{E}+00$ & $7.115 \mathrm{E}-01$ & $7.923 \mathrm{E}-01$ & $1.793 \mathrm{E}+00$ & $7.085 \mathrm{E}-01$ \\
\hline \multirow[t]{5}{*}{$F 14$} & Best & $0.00 E+00$ & $0.00 E+00$ & $1.344 \mathrm{E}+00$ & $8.475 \mathrm{E}+00$ & $2.180 \mathrm{E}+03$ & $1.269 \mathrm{E}+04$ & $1.778 \mathrm{E}+02$ & $7.206 \mathrm{E}+01$ & $2.073 \mathrm{E}+03$ \\
\hline & Median & $1.31 \mathrm{E}-11$ & $0.00 E+00$ & $2.815 \mathrm{E}+01$ & $4.501 \mathrm{E}+01$ & $7.595 \mathrm{E}+03$ & $3.006 \mathrm{E}+04$ & $3.536 \mathrm{E}+02$ & $1.455 \mathrm{E}+03$ & $4.076 \mathrm{E}+03$ \\
\hline & Worst & 5.71E-05 & 2.58E-09 & $1.918 \mathrm{E}+02$ & $7.523 \mathrm{E}+02$ & $3.698 \mathrm{E}+04$ & $5.492 \mathrm{E}+04$ & $7.081 \mathrm{E}+02$ & $6.457 \mathrm{E}+03$ & $9.651 \mathrm{E}+03$ \\
\hline & Mean & 4.10E-06 & 5.08E-11 & $4.898 \mathrm{E}+01$ & $1.695 \mathrm{E}+02$ & $9.522 \mathrm{E}+03$ & $3.091 \mathrm{E}+04$ & $3.554 \mathrm{E}+02$ & $2.093 \mathrm{E}+03$ & $4.532 \mathrm{E}+03$ \\
\hline & $S D$ & $1.12 \mathrm{E}-05$ & $3.61 \mathrm{E}-10$ & $5.028 \mathrm{E}+01$ & $2.220 \mathrm{E}+02$ & $7.144 \mathrm{E}+03$ & $1.079 \mathrm{E}+04$ & $1.311 \mathrm{E}+02$ & $1.975 \mathrm{E}+03$ & $1.861 \mathrm{E}+03$ \\
\hline \multirow[t]{5}{*}{$F 15$} & Best & $0.00 E+00$ & $0.00 E+00$ & $4.499 \mathrm{E}+01$ & $1.382 \mathrm{E}+04$ & $3.980 \mathrm{E}+07$ & $6.110 \mathrm{E}+07$ & $1.542 \mathrm{E}+08$ & $1.474 \mathrm{E}+06$ & $1.283 \mathrm{E}+05$ \\
\hline & Median & $0.00 E+00$ & $0.00 E+00$ & $3.283 \mathrm{E}+02$ & $4.133 \mathrm{E}+05$ & $7.507 \mathrm{E}+07$ & $8.407 \mathrm{E}+07$ & $3.709 \mathrm{E}+08$ & $1.061 \mathrm{E}+07$ & $2.388 \mathrm{E}+05$ \\
\hline & Worst & $0.00 E+00$ & $0.00 E+00$ & $2.518 \mathrm{E}+03$ & $1.247 \mathrm{E}+07$ & $1.557 \mathrm{E}+08$ & $1.111 \mathrm{E}+08$ & $6.179 \mathrm{E}+08$ & $8.335 \mathrm{E}+07$ & $3.528 \mathrm{E}+05$ \\
\hline & Mean & $0.00 E+00$ & $0.00 E+00$ & $4.926 \mathrm{E}+02$ & $1.929 \mathrm{E}+06$ & $8.307 \mathrm{E}+07$ & $8.696 \mathrm{E}+07$ & $3.760 \mathrm{E}+08$ & $1.803 \mathrm{E}+07$ & $2.392 \mathrm{E}+05$ \\
\hline & $S D$ & $0.00 E+00$ & $0.00 E+00$ & $5.304 \mathrm{E}+02$ & $3.115 \mathrm{E}+06$ & $3.129 \mathrm{E}+07$ & $1.489 \mathrm{E}+07$ & $1.192 \mathrm{E}+08$ & $2.213 \mathrm{E}+07$ & $6.522 \mathrm{E}+04$ \\
\hline \multirow[t]{5}{*}{ F16 } & Best & $0.00 E+00$ & $3.65 \mathrm{E}+00$ & $3.462 \mathrm{E}-02$ & $6.731 \mathrm{E}+00$ & $1.609 \mathrm{E}+01$ & $9.950 \mathrm{E}+00$ & $1.112 \mathrm{E}+01$ & $1.029 \mathrm{E}-01$ & $1.271 \mathrm{E}+01$ \\
\hline & Median & $0.00 E+00$ & $1.21 \mathrm{E}+01$ & $3.239 \mathrm{E}+00$ & $1.505 \mathrm{E}+01$ & $2.714 \mathrm{E}+01$ & $1.437 \mathrm{E}+01$ & $1.404 \mathrm{E}+01$ & $7.002 \mathrm{E}-01$ & $1.522 \mathrm{E}+01$ \\
\hline & Worst & $0.00 E+00$ & $1.56 \mathrm{E}+01$ & $2.046 \mathrm{E}+01$ & $2.832 \mathrm{E}+01$ & $3.970 \mathrm{E}+01$ & $1.846 \mathrm{E}+01$ & $1.834 \mathrm{E}+01$ & $4.277 \mathrm{E}+00$ & $1.912 \mathrm{E}+01$ \\
\hline & Mean & $0.00 E+00$ & $1.19 \mathrm{E}+01$ & $3.716 \mathrm{E}+00$ & $1.647 \mathrm{E}+01$ & $2.690 \mathrm{E}+01$ & $1.433 \mathrm{E}+01$ & $1.452 \mathrm{E}+01$ & $1.305 \mathrm{E}+00$ & $1.528 \mathrm{E}+01$ \\
\hline & $S D$ & $0.00 \mathrm{E}+00$ & $2.42 \mathrm{E}+00$ & $4.409 \mathrm{E}+00$ & $5.854 \mathrm{E}+00$ & $6.382 \mathrm{E}+00$ & $2.381 \mathrm{E}+00$ & $1.746 \mathrm{E}+00$ & $1.157 \mathrm{E}+00$ & $1.682 \mathrm{E}+00$ \\
\hline \multirow[t]{5}{*}{ F17 } & Best & $5.63 \mathrm{E}+01$ & $5.47 \mathrm{E}+01$ & $2.719 \mathrm{E}+01$ & $3.067 \mathrm{E}+01$ & $8.767 \mathrm{E}+00$ & $2.230 \mathrm{E}+01$ & $2.157 \mathrm{E}+01$ & $2.534 \mathrm{E}+00$ & $1.483 \mathrm{E}+01$ \\
\hline & Median & $6.18 \mathrm{E}+01$ & $6.40 \mathrm{E}+01$ & $3.085 \mathrm{E}+01$ & $3.181 \mathrm{E}+01$ & $3.105 \mathrm{E}+01$ & $2.809 \mathrm{E}+01$ & $2.788 \mathrm{E}+01$ & $6.883 E+00$ & $2.232 \mathrm{E}+01$ \\
\hline & Worst & $7.51 \mathrm{E}+01$ & $7.12 \mathrm{E}+01$ & $3.320 \mathrm{E}+01$ & $3.270 \mathrm{E}+01$ & $3.283 \mathrm{E}+01$ & $3.144 \mathrm{E}+01$ & $2.988 \mathrm{E}+01$ & $1.464 E+01$ & $2.805 \mathrm{E}+01$ \\
\hline & Mean & $6.24 \mathrm{E}+01$ & $6.33 \mathrm{E}+01$ & $3.053 \mathrm{E}+01$ & $3.178 \mathrm{E}+01$ & $2.885 \mathrm{E}+01$ & $2.776 \mathrm{E}+01$ & $2.718 \mathrm{E}+01$ & $7.684 E+00$ & $2.276 \mathrm{E}+01$ \\
\hline & $S D$ & $4.11 \mathrm{E}+00$ & $3.65 \mathrm{E}+00$ & $1.668 \mathrm{E}+00$ & $4.720 \mathrm{E}-01$ & $5.410 \mathrm{E}+00$ & $2.174 \mathrm{E}+00$ & $2.463 \mathrm{E}+00$ & $3.387 E+00$ & $3.374 \mathrm{E}+00$ \\
\hline \multirow[t]{5}{*}{$F 18$} & Best & $3.40 \mathrm{E}+00$ & $8.10 \mathrm{E}+03$ & $1.131 \mathrm{E}+02$ & $1.637 \mathrm{E}+02$ & $5.202 \mathrm{E}+02$ & $3.915 \mathrm{E}+02$ & $2.564 \mathrm{E}+02$ & $2.611 \mathrm{E}+02$ & $2.948 \mathrm{E}+02$ \\
\hline & Median & $4.59 \mathrm{E}+01$ & $2.22 \mathrm{E}+04$ & $1.979 \mathrm{E}+02$ & $2.550 \mathrm{E}+02$ & $6.376 \mathrm{E}+02$ & $7.343 \mathrm{E}+02$ & $3.163 \mathrm{E}+02$ & $3.582 \mathrm{E}+02$ & $3.687 \mathrm{E}+02$ \\
\hline & Worst & $8.61 \mathrm{E}+01$ & $3.90 \mathrm{E}+04$ & $2.686 \mathrm{E}+02$ & $4.179 \mathrm{E}+02$ & $9.585 \mathrm{E}+02$ & $1.137 \mathrm{E}+03$ & $3.596 \mathrm{E}+02$ & $6.594 \mathrm{E}+02$ & $4.154 \mathrm{E}+02$ \\
\hline & Mean & $4.88 E+01$ & $2.22 \mathrm{E}+04$ & $1.959 \mathrm{E}+02$ & $2.651 \mathrm{E}+02$ & $6.776 \mathrm{E}+02$ & $7.305 \mathrm{E}+02$ & $3.168 \mathrm{E}+02$ & $3.621 \mathrm{E}+02$ & $3.627 \mathrm{E}+02$ \\
\hline & $S D$ & $1.83 \mathrm{E}+01$ & $6.72 \mathrm{E}+03$ & $3.767 \mathrm{E}+01$ & $6.899 \mathrm{E}+01$ & $1.252 \mathrm{E}+02$ & $1.644 \mathrm{E}+02$ & $2.533 \mathrm{E}+01$ & $8.306 \mathrm{E}+01$ & $3.236 \mathrm{E}+01$ \\
\hline \multirow[t]{5}{*}{ F19 } & Best & $3.00 \mathrm{E}-05$ & $7.11 \mathrm{E}+03$ & $3.554 \mathrm{E}-01$ & $5.082 \mathrm{E}-01$ & $4.302 \mathrm{E}+02$ & $6.724 \mathrm{E}+02$ & $2.426 \mathrm{E}+01$ & $9.307 \mathrm{E}+00$ & $3.377 \mathrm{E}+00$ \\
\hline & Median & 4.39E- 05 & $1.00 \mathrm{E}+04$ & $1.328 \mathrm{E}+00$ & $5.697 \mathrm{E}+03$ & $9.703 \mathrm{E}+02$ & $9.243 \mathrm{E}+02$ & $4.450 \mathrm{E}+01$ & $1.757 \mathrm{E}+02$ & $5.082 \mathrm{E}+00$ \\
\hline & Worst & $5.88 \mathrm{E}-05$ & $1.43 \mathrm{E}+04$ & $2.357 \mathrm{E}+00$ & $7.542 \mathrm{E}+03$ & $2.292 \mathrm{E}+03$ & $1.277 \mathrm{E}+03$ & $8.646 \mathrm{E}+01$ & $7.373 \mathrm{E}+02$ & $8.308 \mathrm{E}+00$ \\
\hline & Mean & 4.40E-05 & $9.98 \mathrm{E}+03$ & $1.417 \mathrm{E}+00$ & $5.172 \mathrm{E}+03$ & $1.009 \mathrm{E}+03$ & $9.074 \mathrm{E}+02$ & $4.591 \mathrm{E}+01$ & $2.319 \mathrm{E}+05$ & $5.193 \mathrm{E}+00$ \\
\hline & $S D$ & $6.14 E-06$ & $1.54 \mathrm{E}+03$ & $4.826 \mathrm{E}-01$ & $1.981 \mathrm{E}+03$ & $4.147 \mathrm{E}+02$ & $1.325 \mathrm{E}+02$ & $1.265 \mathrm{E}+01$ & $1.940 \mathrm{E}+02$ & $1.241 \mathrm{E}+00$ \\
\hline \multirow[t]{5}{*}{$F 20$} & Best & $0.00 E+00$ & $0.00 E+00$ & $3.861 \mathrm{E}+00$ & $5.453 \mathrm{E}+00$ & $5.735 \mathrm{E}+00$ & $5.296 \mathrm{E}+00$ & $5.729 \mathrm{E}+00$ & $7.870 \mathrm{E}-01$ & $2.769 \mathrm{E}+00$ \\
\hline & Median & $0.00 E+00$ & $0.00 E+00$ & $5.319 \mathrm{E}+00$ & $5.964 \mathrm{E}+00$ & $6.729 \mathrm{E}+00$ & $5.877 \mathrm{E}+00$ & $6.177 \mathrm{E}+00$ & $2.228 \mathrm{E}+00$ & $3.347 \mathrm{E}+00$ \\
\hline & Worst & $0.00 E+00$ & $0.00 E+00$ & $6.766 \mathrm{E}+00$ & $6.693 \mathrm{E}+00$ & $7.411 \mathrm{E}+00$ & $6.838 \mathrm{E}+00$ & $6.674 \mathrm{E}+00$ & $3.680 \mathrm{E}+00$ & $3.761 \mathrm{E}+00$ \\
\hline & Mean & $0.00 E+00$ & $0.00 E+00$ & $5.262 \mathrm{E}+00$ & $6.019 \mathrm{E}+00$ & $6.617 \mathrm{E}+00$ & $5.946 \mathrm{E}+00$ & $6.187 \mathrm{E}+00$ & $2.064 \mathrm{E}+00$ & $3.324 \mathrm{E}+00$ \\
\hline & $S D$ & $0.00 E+00$ & $0.00 E+00$ & $7.905 \mathrm{E}-01$ & $3.268 \mathrm{E}-01$ & $5.041 \mathrm{E}-01$ & $3.786 \mathrm{E}-01$ & $2.475 \mathrm{E}-01$ & $9.230 \mathrm{E}-01$ & $2.582 \mathrm{E}-01$ \\
\hline
\end{tabular}


Table 6: Non-parametric statistical pairwise comparison results of ABaOA

\begin{tabular}{l|c|c|c|c|c|c|c|c}
\hline \multicolumn{1}{c|}{ ABaOA vs } & BaOA & dBA & BA & PSO & HS & CS & GA & DE \\
\hline Wins/Loses & $14 / 1^{\mathrm{a}}$ & $17 / 3^{\mathrm{a}}$ & $18 / 1^{\mathrm{a}}$ & $19 / 1^{\mathrm{a}}$ & $16 / 3^{\mathrm{a}}$ & $17 / 3^{\mathrm{a}}$ & $16 / 3^{\mathrm{a}}$ & $19 / 1^{\mathrm{a}}$ \\
\hline Sign test p-value & 0.001 & 0.003 & $7.6 \mathrm{E}-05$ & $4.0 \mathrm{E}-05$ & 0.004 & 0.003 & 0.004 & $4.0 \mathrm{E}-05$ \\
\hline Wilcoxon test $p$-value & 0.001 & 0.007 & $\begin{array}{c}2.93 \mathrm{E}- \\
04\end{array}$ & $\begin{array}{c}1.89 \mathrm{E}- \\
04\end{array}$ & 0.002 & 0.001 & 0.003 & $\begin{array}{c}2.93 \mathrm{E}- \\
04\end{array}$ \\
\hline
\end{tabular}

${ }^{\mathrm{a}}$ Level of significant $\alpha=0.05$.

\section{Conclusions and Recommendations}

In this study, the Base Optimization Algorithm (BaOA) which based on combination of basic arithmetic operators is adapted with two displacement parameters and two mutation operators. The introduced algorithm; Bi-Attempted Base Optimization Algorithm (ABaOA), explores the search space through the global optimum by using these two displacement parameters and two basic arithmetic operators only. The performance of $\mathrm{ABaOA}$ examined on twenty well-known classical benchmark functions. Experimental results are statistically analyzed with seven well-known metaheuristic algorithms by using Friedman and Wilcoxon non-parametric statistical comparisons. The statistical tests show that the introduced algorithm outperforms than the other metaheuristic algorithms and it is statistically significant. The $\mathrm{ABaOA}$ is a simple, quick and feasible search approach for both uni-modal and multi-modal numerical functions. When the computational complexity and the speed are the key point for an optimization, the $\mathrm{ABaOA}$ may be an alternative method for numerical optimization problems.

\section{References}

Bednár, D., Lištjak, M., Slimák, A., \& Nečas, V. (2019). Comparison of deterministic and stochastic methods for external gamma dose rate calculation in the decommissioning of nuclear power plants. Annals of Nuclear Energy, 134, 67-76.

Campbell, S. D., Sell, D., Jenkins, R. P., Whiting, E. B., Fan, J. A., \& Werner, D. H. (2019). Review of numerical optimization techniques for meta-device design. Optical Materials Express, 9(4), 1842-1863.

Cao, Y., Lu, Y., Pan, X., \& Sun, N. (2019). An improved global best guided artificial bee colony algorithm for continuous optimization problems. Cluster computing, 22(2), 3011-3019.

Chakri, A., Khelif, R., Benouaret, M., \& Yang, X. S. (2017). New directional bat algorithm for continuous optimization problems. Expert Systems with Applications, 69, 159-175.

de Melo, V. V., \& Banzhaf, W. (2018). Drone squadron optimization: a novel self-adaptive algorithm for global numerical optimization. Neural Computing and Applications, 30(10), 31173144.

Deb, K., \& Padhye, N. (2014). Enhancing performance of particle swarm optimization through an algorithmic link with genetic algorithms. Computational Optimization and Applications, 57(3), 761-794.

Dorigo, M., \& Blum, C. (2005). Ant colony optimization theory: A survey. Theoretical computer science, 344(2-3), 243278.

Holland, J. H. (1962). Outline for a logical theory of adaptive systems. Journal of the ACM (JACM), 9(3), 297-314.
Karaboga, D., \& Akay, B. (2009). A comparative study of artificial bee colony algorithm. Applied mathematics and computation, 214(1), 108-132.

Liberti, L., \& Kucherenko, S. (2005). Comparison of deterministic and stochastic approaches to global optimization. International Transactions in Operational Research, 12(3), 263-285.

Song, Y., Wang, F., \& Chen, X. (2019). An improved genetic algorithm for numerical function optimization. Applied Intelligence, 49(5), 1880-1902.

Xing, B., \& Gao, W. J. (2014). Innovative computational intelligence: a rough guide to 134 clever algorithms (Vol. 62, pp. 22-28). Cham: Springer international publishing.

Yadav, A., Sadollah, A., Yadav, N., \& Kim, J. H. (2020). Selfadaptive global mine blast algorithm for numerical optimization. Neural Computing and Applications, 32(7), 24232444.

Zang, W., Ren, L., Zhang, W., \& Liu, X. (2018). A cloud model based DNA genetic algorithm for numerical optimization problems. Future Generation Computer Systems, 81, 465477. 